\title{
Analysis of mode III interlaminar fracture toughness of laminated composites using a novel testing device
}

\author{
López-Menéndez, A. ${ }^{a}$, Viña, J. ${ }^{a^{*}}$, Argüelles, A. ${ }^{b}$, Viña, I. ${ }^{b}$, Rubiera, S. ${ }^{b}$ \\ a Department of Materials Science and Metallurgical Engineering, University of Oviedo, \\ Campus Universitario, 33203 Gijón, Asturias, Spain \\ ${ }^{b}$ Department of Construction and Manufacturing Engineering, University of Oviedo, \\ Campus Universitario, 33203 Gijón, Asturias, Spain
}

\section{Abstract.}

The delamination phenomenon is one of the major failure mechanisms in composite materials formed by the stacking of successive layers. The fracture mode that has been studied the least within this phenomenon is mode III, due to the difficulty its simulation involves. A novel test device is presented in this paper that allows laminated composites to be subjected to this mode of loading. Characterization tests were carried out on two materials with different matrices in order to analyse the influence of the type of matrix in the fracture behaviour under mode III-fracture. Furthermore, two analytical models adapted to this test methodology have been used for the determination of the value of the energy release rate for this mode of loading in both materials. The load was applied at different distances from the front of the notch, this being another parameter that was analysed.

From the results obtained it follows that the device used in this work allows a dominant mode III and that this result holds for different points of load application studied.

Keywords: mode III, delamination, fracture

\section{Introduction}

In a laminated composite material, in which the laminae that compose it are considered practically homogenous, the main damage macromechanisms are the initiation and propagation of cracks between layers. Separation along the interface of the layers of material is known as delamination.

The growth of delamination leads to a marked reduction in the strength and rigidity of the element, which can produce the collapse and catastrophic failure of the structure. Crack propagation can occur in three failure modes. This paper focuses on mode III 
failure, also known as tearing mode failure, when the shear stress moves the lips of the crack in a parallel direction to the crack front, in perpendicular to crack growth [1].

Numerous papers have been published on this fracture mode using different loading devices.

The Split Cantilever Beam (SCB) test [2-5] uses beam-shaped specimens, employed in DCB and ENF tests. The load is applied to this specimen parallel to the delamination plane in a uniaxial test. Good results were obtained using this test, although induced irregular overloads appear. After several partial modifications, Szekrényes [6] introduced substantial modifications in the test method, giving rise to the MSCB. In this case, two loading systems are applied to the test specimen. As in the SCB test, the dominant load is applied at the end of the specimen parallel to the delamination plane, while a second system of loads is applied in the zone of the insert with the aim of preventing torsion of the specimen.

The Edge Crack Torsion (ECT) test [7-12] supposed an evolution in and significant contribution to the study of interlaminar fracture under mode III loading, being the most widely used of all tests for this type of loading. It is based on the application of a torque at the embedded end of the specimen, or a torque at both ends of the specimen.

Another of the methods that have been used is the Anti-Clastic Plate Bending (ACPB) test, initially developed by Farshad and Flüeler [13] to calculate the shear elastic module of materials. This method employs a rectangular specimen with two lateral notches along its midline, one on each side of the specimen, with the aim of producing pure shear by means of a bending test.

Yoshikara [14] developed the method known as the Four Point Bend End-Notched Flexure (4-ENF ${ }_{\text {III }}$ ) test, which is a mode III variation of the $4 E N F$, which was initially devised to calculate the R-curves of wood. A large-sized, complex-shaped specimen is used in this test, which does not achieve more than $90 \%$ of mode III failure.

A new test method (6ECT) has recently been developed based on modifications to the conventional ECT method [15]. This method allows the determination of R-curves under mode III failure. Studies carried out applying this method report that the energy release rate $\left(G_{I I I}\right)$ increases with increasing crack length.

Finally, Davidson and Sediles [16] introduced a new test method called the ShearTorsion-Bending (STB) test that allows testing the resistance to delamination under any kind of mixed mode loading, which was subsequently modified to study mode III fracture. The loading device requires excessive complexity due to the possibility of testing under the three loading modes simultaneously.

\section{Test device}


A valid test device has been designed to cause delamination of the composite under pure mode III fracture, which is not achieved with the other aforementioned SCB, MSCB and ECT devices.

The device (Figure 1) consists of a T-shaped part (6) which has a prismatic groove machined along the entire length of its upper face with the exact dimensions of the width and of half the thickness of the test specimen (5). The way in which this element is designed means that it can be attached to the test machine in different positions by means of the clamp (2), which is in turn fixed to the frame of the test machine.

Figure 1. Test device and regulation of the loading point on the specimen

The load is applied to the test specimen via a load-block (7) fixed to a linear bearing (4) that moves along a guide rail. The guide rail is fixed to the clamp (1), which is in turn coupled to the actuator of the test machine. The load-block includes two small cylinders of $2 \mathrm{~mm}$ in diameter (8) located one on either side of the specimen which are responsible for transmitting the load. These cylinders act tangentially on the specimen, ensuring a point load on its upper half (upper lip).

By means of this arrangement of elements, it is possible to regulate the point of application of the load on the specimen by simply sliding the load block (7) along the longitudinal guide rail (3), making the cylinder (8) which transmits the load coincide at the desired point " $\mathrm{a}_{0}$ " (Figure 1). In addition, any possible warping of the specimen is restricted during the test. The combination of the linear bearing and the precise way of applying the load ensures that the load is always transmitted perpendicular to the specimen via the angular displacement of the actuator. Furthermore, the shape and dimensions of the load-block (7) prevent rotation of the specimen with respect to its longitudinal axis.

Figure 2 shows a photograph of the device installed on the test equipment, in which the fundamental components can be seen.

Figure 2. Test device

\section{Analytical formulation}

For the calculation of the energy release rate, under mode III fracture a classical methodology based in the compliance method

$$
\left.\frac{\mathrm{CP}^{2}}{2 \mathrm{~b}} \frac{\mathrm{dC}}{\mathrm{dL}}\right|_{\mathrm{L}=a}
$$


The geometry of the specimen used is shown in figures 3 and 4 .

Figure 3. Zone of initial delamination.

For adaptation of the classical formulation, has been considered that the device used restricts the transverse movement and the turns of the lower half of the specimen and that the effect of shear may be representative. So the effects of bending moment and shear generated during loading of the specimen were considered for calculating the transverse displacement of the upper half of the specimen. Moreover, two different formulations for calculating the displacement of the point of load application were used in order to analyse the possible influence of warping of the straight sections of the specimen during the loading: Timoshenko short beams (considering warpage of the section) and the Classical theory of bending for long beams (without warping of the section). It should be noted that the device and test equipment used allows the application of some axial load which limits, significantly, the possibility of warping of the straight sections in the tip of delamination and along its length.

The energy release rates used are:

According to the Timoshenko beam theory:

$$
G=\frac{1}{b}\left(\frac{\mathrm{P}^{2} \mathrm{~L}^{2}}{2 \mathrm{EI}_{\mathrm{z}}}+\frac{3 \mathrm{P}^{2}}{4 \mathrm{bhG}}\right)
$$

while according to the other theory, it will be:

$$
\mathrm{G}=\frac{1}{\mathrm{~b}}\left(\frac{\mathrm{P}^{2} \mathrm{~L}^{2}}{2 \mathrm{EI}_{\mathrm{z}}}+\frac{\mathrm{P}^{2} \mathrm{~b}^{2}}{20 \mathrm{GI}_{\mathrm{z}}}\right)
$$

\section{Experimental procedure}

Two types of composite materials were used. One of the materials is made from Hexply ${ }^{\circledR} 8552$ prepreg epoxy resin tape as the matrix and HexTow ${ }^{\circledR}$ AS4 high resistance and damage tolerance unidirectional carbon fibre reinforcement, marketed under the name Hexply ${ }^{\circledR}$ AS4/8552 RC34 AW196. The other material is made from Hexply ${ }^{\circledR} 3501$ 6 prepreg epoxy resin tape as the matrix and high strength and HexTow ${ }^{\circledR}$ AS4 high resistance and damage tolerance unidirectional carbon fibre reinforcement, marketed under the name Hexply ${ }^{\circledR}$ AS4/3501-6 RC37 AW190.

DCB specimens were used for the static characterization of delamination under mode III fracture. These specimens are made from a carbon fibre laminated composite with unidirectional reinforcement. They are uniform rectangular specimens, of thickness $h$ and width $\mathrm{b}$, with a non-adherent artificial crack initiator of length $a_{\mathrm{i}}$ inserted in the mid-plane that serves to initiate delamination (Figure 4). 
Figure 4. Geometry of the Longitudinal Half Fixed Beam (LHFB) specimen with the inserted embedded longitudinally in its lower lip.

The laminate used in the tests was symmetrical, with an even number of unidirectional layers. The laminate is composed of 32 plies at an orientation of $0^{\circ}$ with respect to the carbon fibre reinforcement, in which an anti-adherent insert was placed in its midplane during the manufacturing process, thus obtaining the aforementioned configuration: 16 plies at $0^{\circ}$ insert/ 16 plies at $0^{\circ}$. The dimensions of the specimens used in the tests were:

- Length (L): 160-200 mm

- Width (b): $10 \mathrm{~mm}$

- Thickness (h): $6 \mathrm{~mm}$

- Length of initial delamination $\left(a_{0}\right): 1-30 \mathrm{~mm}$

- Total length of the crack initiation insert $\left(a_{i}\right): 55-60 \mathrm{~mm}$

The device used was a $w+b$ servohydraulic machine, with a $100 \mathrm{kN}$ load cell and a $500 \mathrm{Nm}$ torque cell, controlled by software. The tests were conducted under angle control (displacement control).

\section{Results and discussion}

The tests were performed on specimens with different initial crack lengths, $a_{0}: 1 \mathrm{~mm}$, $5 \mathrm{~mm}, 10 \mathrm{~mm}, 15 \mathrm{~mm}, 20 \mathrm{~mm}, 25 \mathrm{~mm}$ and $30 \mathrm{~mm}$, for each of the two materials AS4/8552 and AS4/3501-6.

The static tests under mode III loading were carried out as per the previously described procedure. The resistance to interlaminar fracture under mode III loading was calculated using the aforementioned theories, namely the Timoshenko beam theory for the behaviour of short beams (considering warping of the section) and the EulerBernoulli or classical beam theory for the bending of long beams (without warping of the section).

The results for the energy release rate, $G_{I I I}$, are shown below for different initial crack lengths, $a_{0}$, according to the type of material used to manufacture the specimens. The maximum load point, $\mathrm{P}_{\mathrm{MAX}}$, was taken into account to calculate the energy release rate. Table 1 shows the mean values of the energy release rate, $G_{\| I c}$, for different initial delamination lengths, $a_{0}$, according to the Timoshenko beam theory (for short beams).

Table 1. Mean values of $G_{\text {III }}$ as a function of the initial crack length for both materials according to the Timoshenko beam theory. 
The values of the above table are represented in Figure 5. The graph shows that $\mathrm{G}_{\text {IIIC }}$ is visibly higher for specimens with an initial crack length of $1 \mathrm{~mm}$, stabilizing asymptotically at a constant value for crack sizes between 5 and $30 \mathrm{~mm}$.

Figure 5. Mean $\mathrm{G}_{\text {IIIC }}$ values as a function of the initial crack length of the specimens of materials AS4/8552 and AS4/3501-6, according to the Timoshenko beam theory for short beams.

Similarly, Table 2 shows the mean values of the energy release rate, $G_{\text {IIIc }}$, of the two materials studied here for different lengths of initial delamination, $a_{0}$, according to classical beam theory for the bending of long beams.

Table 2. Mean $G_{\text {III }}$ values as a function of the initial crack length for both materials, according to classical beam theory.

The values of the above table are represented in Figure 6. As occurred with the Timoshenko beam theory, this graph shows that $G_{\text {IIIC }}$ is also visibly higher for specimens with an initial crack length of $1 \mathrm{~mm}$, stabilizing asymptotically at a constant value for crack sizes between 5 and $30 \mathrm{~mm}$.

Figure 6. Mean $G_{\text {IIIC }}$ values as a function of the initial crack length in specimens of materials AS4/8552 and AS4/3501-6, according to classical beam theory for the bending of long beams.

It should also be noted that the lowest energy release rate value was always obtained for the material with the 8552 matrix. This matrix is made with the aim of obtaining a high level of toughness. This has been demonstrated in the case of mode I fracture [17]. However, it has been shown that the desired effect is not achieved under mode II [18-20] or mode III fracture, as demonstrated here.

One of the important issues that were raised during this work was to determine the influence of the field of stresses and strains, assuming elastic behaviour of the material, generated in the front of delamination in the vertical and longitudinal direction. In order to minimize and know its influence have followed different strategies:

On the one hand, it has been considered that the possible shear stress in the longitudinal direction of the laminate would create a relatively important part of mode II. In order to avoid it, the testing device allows free movement of the specimen in the longitudinal direction. Moreover, the possible warping or vertical movement of the 
sample during the test also would create a shear stress. It would cause a certain component in mode I, (separation in the vertical direction from the lips of the specimen). So the designed device avoids this warping in the loading part of the sample while the load in the specimen is applied in a point with a single loading roller.

On the other, a study of the displacement field generated during the testing process was made from the experimental point of view for different specimens and different cracks lengths. This work let to identify the active components of tensor deformation using a video digital image correlation equipment, see figures 7 to 9 .

In order to validate the proposed methodology, based on the consideration that during the tests carried out with the device shown previously (figure 2) the dominant mode of fracture is Mode III, during the tests, from the beginning of the loading process to the complete delamination of the specimen, and synchronously with the loading process was carried out the capture of digital images of the crack tip by a 3D video correlation system in order to determine the displacement field in the tip of the crack, and indirectly dominant mode of fracture. So, in an initial reference instant and later, during the delamination process, the three-dimensional coordinates of the object (figure 7) have been obtained, creating a digital model of mesh in 3D coordinates, allowing to evaluate the displacement field in representative points of the specimen along its thickness and for different lengths of delamination.

Figure 7. Mesh of 3D coordinates (digital model)

Analyzing the displacement field determined, using the explained methodology, the ratios of the different fracture modes for several lengths of delamination were obtained and shown in Figures 8 and 9 for the two materials analyzed AS4 / 8552 and AS4 / 3501-6, respectively.

Figure 8. Ratios of fracture modes for the material AS4/8552

Figure 9. Ratios of fracture modes for the material AS4/3501-6

In summary, from the analysis of displacement field in the fracture zone, the average values of the percentages of the components of each fracture mode of total energy release rate, $\mathrm{G}_{\mathrm{T}}$, for the material $\mathrm{AS} 4 / 8552$ have been: $97.92 \%$ of mode III, $2.02 \%$ of mode II and $0.06 \%$ of mode I, and for the AS4/3501- 6 the percentages obtained were: $98.27 \%$ of mode III, $1.69 \%$ of mode II and $0.04 \%$ of mode I.

\section{Conclusions.}


It can be concluded from the experimental study that the test devices and the methodology developed in this paper to characterize composite materials subjected to the process of delamination under mode III fracture loading are both suitable and ensure initiation of the process under mode III.

The combination of two actuators in the test equipment, one linear and the other angular, provides a high degree of reliability in the results by preventing longitudinal warping of the specimen, while at the same time simplifying the test method compared to other current methodologies.

The type of specimen used (DCB) is suitable for characterizing the material under mode III fracture.

The formulas for calculating the energy release rate under mode III fracture loading applied in this study are considered to provide a valid value for this parameter which is in keeping with those obtained in other research studies on these materials using different test methodologies or by means of numerical modelling.

The most conservative calculation formula and hence the one that is considered more suitable from the point of view of safety and the testing methodology used with restriction of the warping of the straight sections is the one developed from classical beam theory for the bending of long beams.

For both materials, the length of the insert initiating the delamination process does not have a significant influence from a certain value onward. The energy release rate under mode III fracture may hence be considered independent of the crack length employed.

Of the two materials studied in this paper, the one with a more fragile matrix, i.e. AS4/3501-6, is the one that provides higher values of fracture toughness under this loading mode. The same behavior was observed in mode II.

The variations in results are relatively low compared to those usually obtained for carbon fibre-reinforced composite materials.

\section{Acknowledgements}

The authors are grateful for financial funding from Spain's “Ministerio de Economía y Competitividad" through research Project MAT2013-48107-C3-3-R

\section{BIBLIOGRAPHY}


[1] D. Broek, "Elementary Engineering Fracture Mechanics", Kluwer Academic Publishers Group, 4th Edition, 1986.

[2] S.L. Donaldson, "Mode III Interlaminar Fracture Characterization of Composite Materials", Composite Science and Technology 32 (1988) 225-249

[3] S.F. Hwang, C.L. Hu, "Tearing Mode Interlaminar Fracture Toughness of Composite Materials", Polymer Composites 22(1) (2001) 57-64

[4] N.K. Naik, K.S. Reddy, S. Meduri, N.B. Raju, P.D. Prasad, SK.N.M. Azad, P.A. Ogde, B.C.K. Reddy, "Interlaminar Fracture Characterization for Plain Weave Fabric Composites", Journal of Materials Science 37 (2002) 2983-2987.

[5] F. Sharif, M.T. Kortschot, R.H. Martin, "Mode III Delamination using a Split Cantilever Beam" in Martin, R.H. (ed.) Composite Materials fatigue and Fracture- Fifth Volume, ASTM STP 1230, American Society for Testing and Materials, Philadelphia, 8599 (1995).

[6] A. Szekrényes, "Improved Analysis of the Modified Split-Cantilever Beam for Mode III Fracture" International Journal of Mechanical Sciences 51 (2009) 682-693.

[7] J. Li, S.M. Lee, E.W. Lee, T.K. O’Brien “Evaluation of the edge crack torsion test (ECT) for mode III interlaminar Fracture Toughness of Laminated Composites" Journal of Composites Technology and Research 19 (1997) 174-183.

[8] W.C. Liao and C.T. Sun "The Delamination of Mode III fracture Toughness in Thick Composite Laminates" Composites Science and Technology 56 (1996) 489-499.

[9] D. Pennas, W.J. Cantwell and P. Compston "The Influence of Strain Rate on the Mode III Interlaminar Fracture of Composite Materials" Journal of Composite Materials 41 (2007) 2595-2614.

[10] A.B. Morais, A.B. Pereira, M.F.S.F. de Moura and A.G. Magalhaes "Mode III Interlaminar Fracture of Carbon /Epoxy Laminates Using the Edge Crack Torsion (ECT) Test" Composites Science and technology 69 (2009) 670-676.

[11] M. de Moura, M. Fernandez, A. de Morais and R. Campilho "Numerical Analysis of the Edge Crack Torsion Test for Mode III Interlaminar Fracture of Composite Laminates" Engineering Fracture Mechanics 76 (2009) 469-478.

[12] D. Pennas and W. Cantwell "The Influence of Loading rate on the Mode III Interlaminar Fracture Toughness of Composite/Steel Bi-material Systems" Journal of Composite Materials 43 (2009) 2255-2268.

[13] M. Farshad and P. Flüeler "Investigation of Mode III Fracture Toughness using Anti-Clastic Plate Bending Method" Engineering Fracture Mechanics 60 (1996) 597603.

[14] H. Yoshihara "Examination of the 4-ENF Test for Measuring the Mode III R-Curve of Wood" Engineering Fracture Mechanics 73 (2006) 42-63. 
[15] A. Pereira, A. de Morais and M. de Moura "Design and Analysis of a New Six-Point Edge Crack Torsion (6ECT) Specimen for Mode III Interlaminar Fracture Characterization" Composites Part A 42 (2011) 131-139.

[16] B.D. Davidson and F.O. Sediles "Mixed-Mode I-II-III Delamination Toughness Determination via a Shear-Torsion-Bending Test" Composites Part A 42 (2011) 589603.

[17] A. Argüelles, J. Viña, A.F. Canteli, A. Lopez "Influence of the Matrix Type on the Mode I Fracture of Carbon-Epoxy Composites Under Dynamic Delamination" Experimental Mechanics 51 (2011) 293-301.

[18] A. Argüelles, J. Viña, A.F. Canteli, J. Bonhomme "Influence of Resin Type on the Delamination Behavior of Carbon Fiber Reinforced Composites Under Mode-II Loading" International Journal of Damage Mechanics 20 (2011) 963-978.

[19] V. Mollón, J. Bonhomme, J. Viña, A. Argüelles, A. Fernández-Canteli "Influence of the principal tensile stresses on delamination fracture mechanisms and their associated morphology for different loading modes in carbon/epoxy composites" Composites Part B: Engineering, 43 (2012) 1676-1680.

[20] S. Alessi, G. Pitarresi, G. Spadaro "Effect of hydrothermal ageing on the termal and delamination fracture behaviour of CFRP composites" Composites Part B: Engineering, 67 (2014) 145-153. 